\title{
Analysis of materials systems represented by graphs
}

DOI:

10.1007/978-3-319-91989-8_56

\section{Document Version}

Accepted author manuscript

Link to publication record in Manchester Research Explorer

\section{Citation for published version (APA):}

Jivkov, A. (2018). Analysis of materials systems represented by graphs. In Proceedings of the 1st International Conference on Theoretical, Applied, Experimental Mechanics (pp. 249-254) https://doi.org/10.1007/978-3-31991989-8_56

\section{Published in:}

Proceedings of the 1st International Conference on Theoretical, Applied, Experimental Mechanics

\section{Citing this paper}

Please note that where the full-text provided on Manchester Research Explorer is the Author Accepted Manuscript or Proof version this may differ from the final Published version. If citing, it is advised that you check and use the publisher's definitive version.

\section{General rights}

Copyright and moral rights for the publications made accessible in the Research Explorer are retained by the authors and/or other copyright owners and it is a condition of accessing publications that users recognise and abide by the legal requirements associated with these rights.

\section{Takedown policy}

If you believe that this document breaches copyright please refer to the University of Manchester's Takedown Procedures [http://man.ac.uk/04Y6Bo] or contact uml.scholarlycommunications@manchester.ac.uk providing relevant details, so we can investigate your claim.

\section{OPEN ACCESS}




\title{
Analysis of materials systems represented with graphs
}

\author{
Andrey P Jivkov \\ ${ }^{1}$ Mechanics and Physics Research Group, School of Mechanical, Aerospace and Civil Engi- \\ neering, The University of Manchester, Oxford Road, Manchester M13 9PL, UK \\ andrey.jivkov@manchester.ac.uk
}

\begin{abstract}
Presented is a rigorous mathematical formulation of boundary value problems defined on discrete systems described by mathematical graphs. The formulation is applicable to mechanical and physical problems and includes an effective algebraic framework and efficient computational implementation. Mechanical problems involving damage initiation and evolution are soled to illustrate the proposed method. It is concluded that the graph-theoretical approach to discrete systems offers substantial benefits in terms of conceptual clarity and computational efficiency.
\end{abstract}

Keywords: Discrete analysis, Boundary value problems, Damage and failure

\section{Introduction}

Representing materials sub-continuum structures with mathematical graphs, or 1complexes as they are known in algebraic topology, takes inspiration from the classical mechanics of atom interactions. A specific structure, e.g. a set of grains forming a polycrystalline material, which can be generally represented with a 3-complex with cells, faces, edges and vertices, is simplified or reduced to a 1-compex (a graph) by a problem-specific selection of vertices or sites and edges or bonds. There is a considerable body of research where beams $[1,2]$ and springs $[3,4]$ have been investigated as bonds in order to capture the mechanical response of materials at different scales.

The attraction of graph-based, also known as lattice, models is their discrete nature, which allows for modifying the behavior of individual bonds or sets of bonds to capture heterogeneity at microstructural level, e.g. by incorporating measurable features of variable sizes, such as pores or particles, and measurable properties of variable magnitudes, such as elastic constants or grain boundary excess energies. Further, specifically for deformation and fracture analysis, the discrete representation allows for capturing explicitly micro-crack initiation, growth and coalescence through bond deletion. It is worth noting that lattice models are not limited to mechanics but have also been used for analysis of heat and mass transfer in solids [5, 6], and transport through porous media [7, 8]. Finally, the graph-based approach offers substantial computational efficiency compared to representations with 3-complexes, which can also incorporate spatially distributed features and properties, but at this stage require substantial new developments for analysis of damage evolution. 
The aim of this work is to present a rigorous mathematical description of graphbased models of materials structures and their analysis, applicable to any field of study. Proposed is a new formulation of boundary value problems on graphs and associated efficient numerical approach for construction and analysis of such problems. This is implemented in a $\mathrm{C}++$ library capable of tracking topological and geometric modifications arising from various physical and mechanical problems. Numerical examples illustrating the benefits of the proposed framework are given at the end.

\section{Analysis on graphs}

\subsection{Mathematical basis - graph topology}

An abstract graph $G=(V, E)$ is a tuple of finite sets $V$ and $E$, where elements $e_{i} \in E$, called edges, are pairs of elements $v_{j} \in V$, called vertices. For the purposes of this work we consider only graphs, for which elements of $E$ are unordered and distinct pairs $\left(v_{j}, v_{k}\right)$ with $j \neq k$, i.e. undirected graphs without multiple edges and loops. The number of vertices is given by $|V|=n$ and edges by $|E|=m$.

A graph from the class considered here can be equipped with orientation by specifying an arbitrary but fixed order of the vertices of each edge $e_{i}=\left(v_{j}, v_{k}\right) \in E$, e.g. by selecting $v_{j}$ to be the first vertex (origin, denoted by $\left.v_{j}=o\left(e_{i}\right)\right)$ and $v_{k}$ to be the second vertex (terminus, denoted by $v_{k}=t\left(e_{i}\right)$ ), the oriented edge is $e_{i}=\left[v_{j}, v_{k}\right]$.

The topology of an oriented graph can be encoded into a $m \times n$ matrix $\mathbf{A}$, referred to as the incidence matrix, with the following components

$$
a_{i j}=\left\{\begin{array}{c}
+1, \text { if } v_{j}=t\left(e_{i}\right) \\
-1, \text { if } v_{j}=o\left(e_{i}\right) . \\
0, \text { otherwise }
\end{array}\right.
$$

The significance of the incidence matrix cannot be overemphasized, as together with the graph topology it provides the boundary operator on nodes, while its transpose provides the boundary operator on edges. Specifically, for a discrete function over nodes $\boldsymbol{u}$, the boundary operator gives a discrete function over edges $\mathbf{A} \boldsymbol{u}$, computing the differences between nodal values at edges, hence a discrete gradient. Inversely, for a discrete function over edges $\boldsymbol{f}$, the transposed boundary operator gives a discrete function over nodes $\mathbf{A}^{\boldsymbol{T}} \boldsymbol{f}$, computing the algebraic sums of edge values at nodes, hence a discrete divergence. Thus the incidence matrix establishes the basis for analysis on graphs.

\subsection{Geometry and physics - graph metric}

Formulation of mechanical or physical problems on graphs requires a geometric realisation of $G$, i.e. embedding the graph into a metric space, for example $\mathbb{R}^{d}$, by associating a point (coordinates) $\mathbf{x}_{j} \in \mathbb{R}^{d}$ with every vertex $v_{j} \in V$. If the edges are considered as straight segments described by $\mathbf{y}_{\boldsymbol{i}}=\lambda \mathbf{x}_{j}-(1-\lambda) \mathbf{x}_{k}, \lambda \in[0,1]$, the embedding induces a standard graph metric, given by edge lengths $l_{i}=\left\|\mathbf{x}_{\boldsymbol{j}}-\mathbf{x}_{\boldsymbol{k}}\right\|$ for 
$e_{i}=\left[v_{j}, v_{k}\right]$. It is required that the embedding does not lead to vertices in the interior of edges, i.e. $\mathbf{x}_{l} \neq \lambda \mathbf{x}_{j}-(1-\lambda) \mathbf{x}_{k}$, for $\lambda \in(0,1)$ and $\forall l$, and to edges of zero size, i.e. $l_{i}>0 \forall i$. In practice the embedding will be dictated by a specific selection of vertices and edges that represents given material system, so the selection should obey these restrictions. In what follows consideration will be given to embedding in $\mathbb{R}^{3}$.

Each edge $e_{i}$ of an embedded graph can be assigned a triad of unit vectors - tangent, $\mathbf{t}_{\boldsymbol{i}}$, normal, $\mathbf{n}_{\boldsymbol{i}}$, and bi-normal, $\mathbf{b}_{\boldsymbol{i}}-$ which can be expressed in local and global coordinate systems by the matrices $\widehat{\mathbf{B}}_{\boldsymbol{i}}$ and $\mathbf{B}_{\boldsymbol{i}}$, respectively, related by $\widehat{\mathbf{B}}_{\boldsymbol{i}}=\mathbf{Q}_{i}^{\mathrm{T}} \mathbf{B}_{\boldsymbol{i}}$ and $\mathbf{B}_{\boldsymbol{i}}=\mathbf{Q}_{i} \widehat{\mathbf{B}}_{i}$, where $\mathbf{Q}_{i}$ is the matrix of direction cosines $\mathbf{Q}_{i} \mathbf{Q}_{i}^{\mathrm{T}}=\mathbf{Q}_{i}^{\mathrm{T}} \mathbf{Q}_{i}=\mathbf{I}_{3 \times 3}$. Further, the incidence matrix is expanded into a $3 m \times 3 n$ matrix $\mathbf{A}$ with components

$$
a_{i j}=\left\{\begin{array}{cl}
+\mathbf{I}_{3 \times 3}, & \text { if } v_{j}=t\left(e_{i}\right) \\
-\mathbf{I}_{3 \times 3}, & \text { if } v_{j}=o\left(e_{i}\right) . \\
\mathbf{0}_{3 \times 3}, & \text { otherwise }
\end{array}\right.
$$

In addition to the geometric embedding, mechanical and physical problems require assignment of edge weights, which represent resistances to the particular phenomenon studied. Clearly they depend on problem-specific intrinsic physical properties, as well as on local geometric characteristics, and are typically specified with respect to the local coordinate system. This requires a weight function $E \rightarrow \mathbb{R}^{3}$ assigning to each edge $e_{i}$ resistances $\left(\alpha_{i}, \beta_{i}, \gamma_{i}\right)$ in the directions of its unit vectors. Given weight function introduces a metric on the graph, described in the local and global coordinate systems, respectively, by the matrices

$$
\widehat{\mathbf{M}}_{i}=\left[\begin{array}{c}
\alpha_{i} \mathbf{t}_{i} \\
\beta_{i} \mathbf{n}_{i} \\
\gamma_{i} \mathbf{b}_{i}
\end{array}\right] \text { and } \mathbf{M}_{i}=\mathbf{Q}_{i} \widehat{\mathbf{M}}_{i} \mathbf{Q}_{i}^{\mathrm{T}} .
$$

It should be noted that for physical problems, such as heat and mass transport, as well as mechanical problems involving spring networks $\alpha_{i} \neq 0, \beta_{i}=\gamma_{i}=0$, while for mechanical problems with more complex interactions $\alpha_{i} \neq 0, \beta_{i}=\gamma_{i} \neq 0$. The full graph metric is given by the block-diagonal matrix $\mathbf{M}=\operatorname{diag}\left(\mathbf{M}_{1}, \mathbf{M}_{2}, \ldots, \mathbf{M}_{m}\right)$.

\subsection{Boundary value problems - graph analysis}

For a given vector-valued function on vertices $\boldsymbol{u}: V \rightarrow \mathbb{R}^{3}$, the product $\mathbf{A} \boldsymbol{u}$ provides the discrete gradient - a vector-valued function on edges $\mathbf{A} \boldsymbol{u}: E \rightarrow \mathbb{R}^{3}$. The graph metric transforms the discrete gradient into flux vector $\boldsymbol{f}=\mathbf{M A} \boldsymbol{u}$, the discrete divergence of which must satisfy equilibrium with external sources, $\boldsymbol{b}$, at nodes, i.e. $\mathbf{A}^{\mathrm{T}} \boldsymbol{f}=\boldsymbol{b}$. This leads to the following expression for the equilibrium of the system

$$
\mathbf{A}^{\mathrm{T}} \mathbf{M A u}=\mathbf{L} \boldsymbol{u}=\boldsymbol{b},
$$

where $\mathbf{L}$ denotes the Laplacian of the weighted graph, which can be shown to be symmetric, positive semi-definite, and singular. 
In order to apply boundary conditions, the degrees of freedom of graph vertices $k=3 n=3|V|$ are sorted into two sets, $k_{1}$ with essential, and $k_{2}$ with natural conditions. This allows for expressing Eq. (4) in the form

$$
\left[\begin{array}{ll}
\mathbf{L}_{11} & \mathbf{L}_{12} \\
\mathbf{L}_{21} & \mathbf{L}_{22}
\end{array}\right]\left(\begin{array}{l}
u_{1} \\
u_{2}
\end{array}\right)=\left(\begin{array}{l}
b_{1} \\
b_{2}
\end{array}\right)
$$

where $\boldsymbol{u}_{\mathbf{1}}$ and $\boldsymbol{b}_{\mathbf{2}}$ collect all components with prescribed values, and $\boldsymbol{u}_{\mathbf{2}}$ and $\boldsymbol{b}_{\mathbf{1}}$ collect all components with unknown values. Notably $\mathbf{L}_{\mathbf{1 2}}=\mathbf{L}_{\mathbf{2 1}}$. Unknowns are found by solving the following two steps

$$
\begin{aligned}
& \mathbf{L}_{22} u_{2}=b_{2}-\mathbf{L}_{21} u_{1}, \\
& b_{1}=L_{11} u_{1}+L_{12} u_{2}
\end{aligned}
$$

Efficient construction of the sub-matrices $\mathbf{L}_{\boldsymbol{i} j}$ can be achieved by splitting the incidence matrix $\mathbf{A}$ into a matrix $\mathbf{U}$ containing only $k_{1}$ columns corresponding to the essential boundary conditions, and a matrix $\mathbf{V}$ containing the remaining $k_{2}$ columns; both matrices have the same rows as $\mathbf{A}$. It can be shown that $\mathbf{L}_{\mathbf{1 1}}=\mathbf{U}^{\mathbf{T}} \mathbf{M U}$, $\mathbf{L}_{\mathbf{2 2}}=\mathbf{V}^{\mathbf{T}} \mathbf{M V}$, and $\mathbf{L}_{\mathbf{1 2}}=\mathbf{V}^{\mathrm{T}} \mathbf{M U}$. Proof and procedure details can be found in [9].

\subsection{Computational implementation}

The algebraic framework for graph analysis described above has been implemented in an in-house code. Of particular interest is to apply the analysis on problems of damage initiation and propagation, which requires handling evolution of the graph topology, e.g. by deleting edges to represent local failures. To this end, a Boost Graph Library (BGL) [10] data structure has been employed to maintain a record of the connectivity information, from which $\mathbf{A}$ and $\mathbf{M}$ can be easily extracted. Therefore, if an edge is to be removed during analysis, the matrices can be output from the data structure once again or modified accordingly. The definition of the physics problem is inherent to the matrix $\mathbf{M}$, therefore minimal user interaction is required to provide the function associated with each edge.

Given a sufficiently large number of vertices and edges, it can be seen that the oriented incidence matrix $\mathbf{A}$ and $\mathbf{M}$ are very sparse. Therefore, a variant of the compressed column storage scheme available in Eigen [11] has been used. Additionally, Eigen is equipped with a number of iterative solvers, of which the conjugate gradient method has been used for tackling the linear form of Eq. (4). For a non-linear Eq. (4), an implementation of Newton's method and Broyden's method [12] has been made.

For a number of problems it is desirable to construct the graph from a set of randomly distributed vertices, with connectivity and other geometric characteristics, potentially required for $\mathbf{M}$, determined by the Voronoi diagram around the vertex set. For such cases the Voro++ [13] has been used to obtain the necessary information. 


\section{Example problems}

Two problems are selected to illustrate the performance of the discrete approach in analysis of damage initiation and fracture: Figures 1 and 2. For simplicity the graphs are made as regular as the geometry allowed and the edges are modeled as trusses, i.e. with graph metric defined by $\alpha_{i}=E A / l_{i}, \beta_{i}=\gamma_{i}=0$, where $E$ and $A$ are the modulus and elasticity and a predefined cross-section (this can be linked to area of the dual face in the Voronoi tessellation if required). Damage initiation and propagation are represented by edge deletion when a critical value of the discrete strain is reached. More details on the procedures used, further examples on physical and mechanical problems and the code developed can be found at [14].
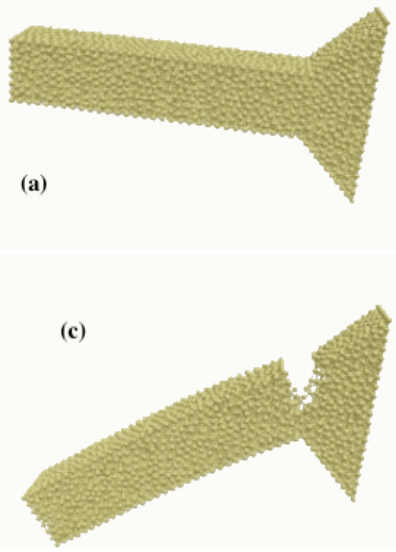
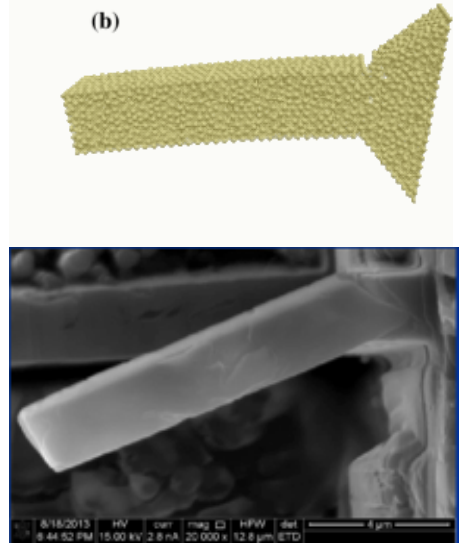

Fig. 1. Process of damage evolution and failure (a)-(c) of graph-modeled beam in comparison with experimentally observed behavior in nuclear graphite micro-cantilever beam (d).

(a)
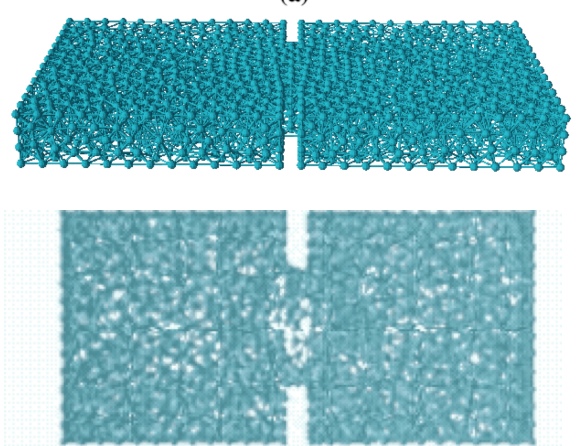

(c)

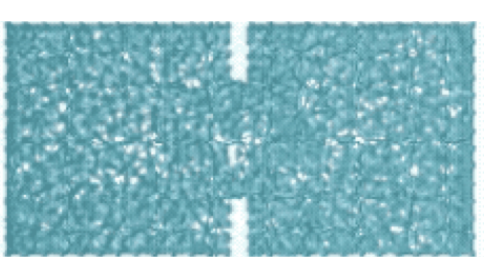

(b)
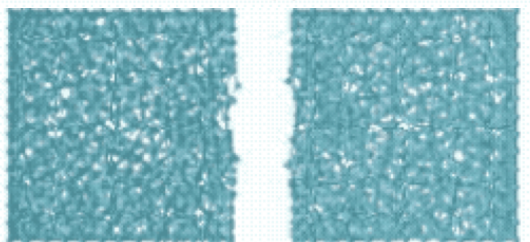

(d)

Fig. 2. Graph model of double edge notched plate (a), and evolution of damage and failure (b)(d) under tension normal to notches. 


\section{Discussion and conclusions}

The results presented demonstrate that the computational model is performing well in terms of capturing the initiation and tracking the propagation of fracture, close to the observed or expected behavior. The graph-theoretical approach offers a clear and concise way for describing discrete systems and allows for computationally efficient algorithms to be employed. Further work is necessary to investigate the applicability of the approach to cases with complex interactions.

\section{Acknowledgments}

The author appreciates highly the financial support of EPSRC via grants EP/K016946/1 "Graphene-based Membranes" and EP/N026136/1 "Geometric Mechanics of Solids".

\section{References}

1. Ostoja-Starzewski, M.: Lattice models in micromechanics. Applied Mechanics Reviews 55(1), 35-60 (2002).

2. Jivkov, A.P., Yates, J.R.: Elastic behaviour of a regular lattice for meso-scale modelling of solids. International Journal of Solids and Structures 49(22), 3089-3099 (2012).

3. Zhang, M., Jivkov, A.P.: Micromechanical modelling of deformation and fracture of hydrating cement paste using X-ray computed tomography characterisation. Composites Part B: Engineering 88, 64-72 (2016).

4. Morrison, C.N., Jivkov, A.P., Vertyagina, Ye., Marrow, T.J.: Multi-scale modelling of nuclear graphite tensile strength using the Site-Bond lattice model. Carbon 100, 273-282 (2016).

5. Feng, Y. T., Han, K., Li, C. F., Owen, D. R. J.: Discrete thermal element modelling of heat conduction in particle systems: Basic formulations. Journal of Computational Physics, 227(10), 5072-5089 (2008).

6. Jivkov, A.P., Yates, J.R.: A discrete model for diffusion-induced grain boundary deterioration. Key Engineering Materials 592-593, 757-760 (2014).

7. Xiong, Q., Jivkov, A.P., Yates, J.R.: Discrete modelling of contaminant diffusion in porous media with sorption. Microporous and Mesoporous Materials 185, 51-60 (2014).

8. Xiong, Q., Joseph, C., Schmeide, K., Jivkov, A.P.: Measurement and modelling of reactive transport in geological barriers for nuclear waste containment. Physical Chemistry Chemical Physics 17, 30577-30589 (2015).

9. Codsi C., Jivkov, A.P.: Mixed boundary value problem for Poisson's equation on weighted graphs with physical application. SIAM Journal on Discrete Mathematics, under review, (2018).

10. Siek, J.G., Lee, L.Q., Lumsdaine, A.: The Boost Graph Library: User Guide and Reference Manual. Portable Documents, Pearson Education (2001).

11. Guennebaud, G., Jacob, B.: Eigen, http://eigen. tuxfamily. org (2010).

12. Broyden, C.G.: A class of methods for solving nonlinear simultaneous equations. Mathematics of computation 19(92), 577-593 (1965).

13. Rycroft, C.H.: VORO++: A three-dimensional Voronoi cell library in C++. Chaos: An Interdisciplinary Journal of Nonlinear Science, 19(4), 041111 (2009).

14. MaPoS Homepage, https://mapos.manchester.ac.uk/, last accessed 31/01/2018 